\title{
Treatment of maternal depression in low-income women: A feasibility study from Kilifi, Kenya
}

\author{
Amber Notiar ${ }^{1}$, Dung Jidong ${ }^{2}$, Florence Hawa ${ }^{1}$, Farah Lunat ${ }^{1}$, Sadia Shah ${ }^{1}$, Paul Bassett ${ }^{1}$, \\ Dawn Edge ${ }^{1}$, Farooq Naeem ${ }^{1}$, and Nusrat Husain ${ }^{1}$ \\ ${ }^{1}$ Affiliation not available \\ ${ }^{2}$ Nottingham Trent University
}

February 5, 2021

\begin{abstract}
Aims of the study: To test the feasibility of a group psychosocial intervention called Learning Through Play (LTP) plus Culturally adapted Cognitive Behaviour Therapy (CaCBT) for depressed Kenyan mothers with children aged 0-36 months. Methods used to conduct the study: This study was a single-arm mixed methods feasibility study to test an integrated parenting intervention for maternal depression in a low-income rural area of Fumbini village in Kilifi District of Kenya. Women between the ages of 18 and 45 years with children up to three years were screened for depression using PHQ-9 (Patient Health Questionnaire). Those scoring above 10 on PHQ-9 were interviewed using the Revised Clinical Interview Schedule (CIS-R) to confirm the diagnosis of depression. Assessments were carried out at baseline and the end of the intervention (3 months), followed by qualitative interviews with 12 women. Qualitative interviews were analysed using thematic analysis from a socialconstructionist theoretical lens. Results of the study: The LTP Plus was both feasible and acceptable with high satisfaction among the participants. Qualitative results showed that the women perceived the intervention as beneficial in reducing the symptoms of depression, coping with stress and negative emotions. The results also indicated that there was a reduction in scores on PHQ-9, GAD-7 with an increase in perceived social support, health-related quality of life and an improvement in mothers' knowledge about child development at the end of the intervention. Conclusions drawn from the study and clinical implications: This study represents the first feasibility research on integrated parenting intervention in Kenya. The results indicated that culturally adapted LTP plus CaCBT is feasible and acceptable in a low-income setting of Kenya. There is now a need to study the clinical and cost-effectiveness of LTP plus CaCBT in an appropriately powered larger randomised control trial, with a longer follow-up period.
\end{abstract}

\section{Hosted file}

Manuscript_Treatment of maternal depression in low-income women.pdf available at https: //authorea.com/users/393831/articles/507366-treatment-of-maternal-depression-in-lowincome-women-a-feasibility-study-from-kilifi-kenya 